\title{
Development and Applicability of Integrative Tumor Response Assays for Metastatic Colorectal Cancer
}

\author{
YONG SIK YOON ${ }^{1,2}$ CHAN WOOK KIM ${ }^{1,2}$, SEON AE ROH ${ }^{2}$, DONG HYUNG CHO ${ }^{2,4}$, \\ TAE WON KIM ${ }^{2,3}$, MOON BO KIM ${ }^{2}$ and JIN CHEON KIM ${ }^{1,2}$ \\ Departments of ${ }^{1}$ Surgery and ${ }^{3}$ Internal Medicine, \\ University of Ulsan College of Medicine, Seoul, Republic of Korea; \\ ${ }^{2}$ Institute of Innovative Cancer Research, Asan Medical Center, Seoul, Republic of Korea; \\ ${ }^{4}$ Graduate School of East-West Medical Science, Kyung Hee University, Gyeonggi-do, Republic of Korea
}

\begin{abstract}
Aim: The present study investigated how well the results of integrative tumor-response assay (ITRA) compared to those of clinical response to chemotherapy in patients with metastatic colorectal cancer (CRC). Patients and Methods: A total of 129 patients with metastatic CRC were prospectively enrolled. ITRA consisted of two sequential histoculture drug-response assays (HDRAs). First-stage HDRAs were performed using 5-fluorouracil with leucovorin and oxaliplatin $(F X)$, or with irinotecan $(F R)$. Second-stage HDRAs (ITRA) were performed for cells surviving after the first-stage HDRA, using $F X, F R$, and their combinations with bevacizumab and cetuximab. Results: Among 129 patients, $42(32.6 \%)$ completed second-line chemotherapy, results that correlated with those of ITRA. The accuracy of ITRA for predicting response to second-line chemotherapy was $61.9 \%$ (26/42), with a sensitivity of $44.4 \%$ (8/18) and a specificity of 75\% (18/24). Conclusion: Despite its relatively low accuracy, ITRA might be a useful technique for predicting therapeutic efficacy and selecting for appropriate first-line and second-line anticancer regimens for patients.
\end{abstract}

Recent trends in cancer treatment involve tailored medicine aimed to maximize individual responsiveness. The drugresponse assay has been developed to increase therapeutic

The contents of this manuscript were previously presented as a poster presentation at the Annual Meeting of The American Association for Cancer Research, New Orleans, Louisiana: 16 to 20 April, 2016.

Correspondence to: Dr. J.C. Kim, Department of Surgery, University of Ulsan College of Medicine, 88, Olympic-ro 43-gil, Songpa-gu, Seoul 05505, Republic of Korea. Tel: +82230103489 , Fax: +82 24749027, e-mail: jckim@amc.seoul.kr

Key Words: Colorectal adenocarcinoma, chemotherapy, histoculture drug-response assay, molecular targeted therapy, treatment outcome. efficacy and reduce anticancer drug toxicity. The histoculture drug-response assay (HDRA) is representative of this effort. It is a methyl thiazolyl-diphenyl-tetrazolium bromide (MTT) assay with the advantage of more correctly reflecting the in vivo microenvironment using three-dimensional multicellular spheroids in tissue culture (1). Previous studies showed 66$92 \%$ accuracy of this assay in terms of correlation with clinical response to chemotherapy for solid tumors, including colorectal cancer (CRC) (2-5).

Approximately $20 \%$ of patients with CRC have stage IV disease. Of these, more than $75 \%$ have unresectable metastases and therefore need palliative treatment $(6,7)$. Most cases are incurable and have gradual progression of disease in spite of palliative chemotherapy. Our previous study demonstrated that HDRA was useful for predicting therapeutic efficacy and selecting the appropriate chemotherapy regimen for patients with metastatic CRC (5). However, an innate limitation of HDRA is that it is an in vitro assay devoid of the tumor microenvironment. Moreover, in cases of recurrent CRC, there is no usable assay to predict sensitivity to second-line chemotherapy.

We developed a novel in vitro chemosensitivity assay based on HDRA, the integrative tumor-response assay (ITRA, Patent No. 10-1235820; the Korean Intellectual Property Office, Seoul, Korea). It simultaneously predicts responsiveness to both first- and second-line chemotherapy. We investigated the chemosensitivity to clinically available regimens by ITRA using primary tumor tissues from patients with metastatic CRC and compared the results with the patients' actual clinical responses.

\section{Patients and Methods}

Patient selection. A total of 129 patients with metastatic CRC were prospectively enrolled. All patients underwent palliative surgery between December 2011 and December 2013. Eligibility criteria included histologically-proven colorectal adenocarcinoma, Eastern Cooperative Oncology Group performance status $\leq 2$, age $\leq 80$ years, 
Table I. Baseline characteristics of patients with metastatic colorectal cancer $(n=120)$.

\begin{tabular}{lc}
\hline Characteristic & No. of patients $(\%)$ \\
\hline Gender, male & $74(61.7)$ \\
Age, years* & $60.6 \pm 11$ \\
Preoperative CEA, $>6 \mathrm{ng} / \mathrm{ml}$ & $60(50.0)$ \\
Location, rectum & $27(22.5)$ \\
Tumor size, $>6 \mathrm{~cm}$ & $47(39.2)$ \\
Histology, mucinous or poorly differentiated & $20(16.7)$ \\
Lymphovascular invasion, yes & $79(65.8)$ \\
Perineural invasion, yes & $57(47.5)$ \\
Mismatch repair defects, yes & $5(4.2)$ \\
Primary tumor resection, yes & $116(96.7)$ \\
\hline
\end{tabular}

CEA, Carcinoembryonic antigen. $*$ Mean \pm SD.

and expectation of palliative chemotherapy. Patients were excluded if they had received preoperative chemotherapy or radiotherapy. We excluded nine cases in which ITRA results were incomplete because of insufficient tumor samples. Baseline demographic and clinicopathological characteristics are described in Table I. The median follow-up period was 28 months (range $=0-64$ months). By the end of the study period, only 42 patients had undergone secondline chemotherapy using regimens that were evaluated with ITRA. The outcomes of those who had both first-line and second-line chemotherapy using tested drug regimens were evaluated for correlation with ITRA results. ITRA results were correlated with clinical response (Figure 1). The study protocol was approved by the Institutional Review Board of Asan Medical Center (Registration No: 2012-0222), in accordance with the Declaration of Helsinki.

Investigated drug combinations and concentrations. The regimens tested included 5-fluorouracil (5-FU) with leucovorin and oxaliplatin (FX), 5-fluorouracil with leucovorin and irinotecan (FR), and these combinations with biologically targeted drugs. First-line chemotherapy regimens were FX or FR and second-line regimens were FX, FR, or one of these combined with a targeted agent (Table II). The targeted drugs used were bevacizumab (Avastin ${ }^{\circledR}$; Roche, Basel, Switzerland) and cetuximab (Erbitux ${ }^{\circledR}$; Merck, Munchen, Germany). The combined regimens were FX with bevacizumab or cetuximab, and FR with bevacizumab or cetuximab. The doses initially used were based on the usual clinical dose as determined from pharmacokinetic and pharmacodynamic parameters obtained through phase I studies and empirical assays $(8,9)$. Median inhibitory concentrations $\left(\mathrm{IC}_{50}\right)$ of tested drugs were determined as those causing a $50 \%$ dose-dependent reduction in cell viability in complete growth medium after $48 \mathrm{~h}$ of continuous exposure to the drug. The cutoff concentrations of the six anticancer drugs used to determine in vitro sensitivity and resistance were $50 \mu \mathrm{g} / \mathrm{ml}$ for 5 FU, $10 \mu \mathrm{g} / \mathrm{ml}$ for leucovorin, $20 \mu \mathrm{g} / \mathrm{ml}$ for irinotecan, $40 \mu \mathrm{g} / \mathrm{ml}$ for oxaliplatin, $20 \mu \mathrm{g} / \mathrm{ml}$ for bevacizumab, and $20 \mu \mathrm{g} / \mathrm{ml}$ for cetuximab, the same as in our previous study of HDRA (5).

Integrative tumor-response assay. ITRA was theoretically based on the HDRA procedure, but in vitro evaluation of resistance to the first-line anticancer drugs was sequentially repeated. In the following description of the procedure, the first three steps for ITRA

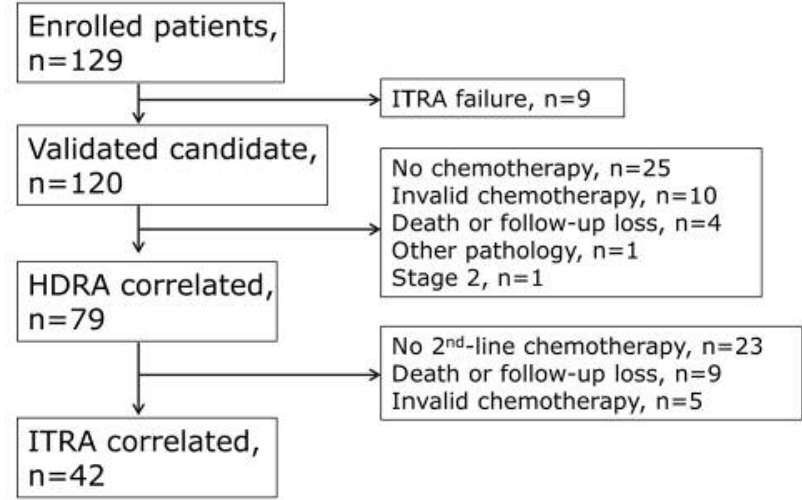

Figure 1. Selection of patients with metastatic colorectal carcinoma. ITRA, Integrative tumor-response assay; HDRA, histoculture drugresponse assay.

Table II. First- and second-line chemotherapy regimens used for patients with metastatic colorectal carcinoma.

\begin{tabular}{|c|c|c|c|}
\hline \multicolumn{2}{|c|}{ First-line } & \multicolumn{2}{|c|}{ Second-line } \\
\hline Therapy & $\begin{array}{l}\text { No. of patients } \\
(\mathrm{n}=79), \mathrm{n}(\%)^{\mathrm{a}}\end{array}$ & Therapy & $\begin{array}{l}\text { No. of patients } \\
(\mathrm{n}=42), \mathrm{n}(\%)^{\mathrm{a}}\end{array}$ \\
\hline \multirow[t]{5}{*}{ FX } & $53(67)$ & FR & $16(38)$ \\
\hline & & FR+bevacizumab & $4(10)$ \\
\hline & & FR+cetuximab & $1(2)$ \\
\hline & & FX+bevacizumab & 0 \\
\hline & & FX+cetuximab & 0 \\
\hline \multirow[t]{5}{*}{ FR } & $26(33)$ & FX & $20(48)$ \\
\hline & & FX+bevacizumab & $1(2)$ \\
\hline & & FX+cetuximab & 0 \\
\hline & & FR+bevacizumab & 0 \\
\hline & & FR+cetuximab & 0 \\
\hline
\end{tabular}

FX, 5-Fluorouracil + leucovorin + oxaliplatin; FR, 5-fluorouracil + leucovorin + irinotecan. aNumber of patients completing chemotherapy.

were similar to those for HDRA. The details for HDRA were the same as in our previous work, except for the tumor volume $(5,10)$. The final two steps were specific for ITRA.

(i) Preparation of tissue samples. At least two sections of tumor tissue $(1 \mathrm{~cm}$ in diameter) were obtained from fresh surgically resected specimens. This was a larger volume than we had previously collected for HDRA only. Tumor samples were aseptically washed in Hank's balanced salt solution (HBSS; Gibco, Gaithersburg, MD, USA). Collagen sponge gels manufactured from pig skin $\left(\right.$ Gelfoam $\left.{ }^{\circledR}\right)$ were purchased from Pharmacia \& Upjohn (Kalamazoo, MI, USA). The cancerous portions of the specimen were minced with scissors into pieces approximately $1 \mathrm{~mm}$ in diameter. Necrotic and non-viable portions were excluded using 3(4,5-dimethylthiazol-2-yl)-5-(3-carboxymethoxyphenyl)-2-(4sulfophenyl)-2-tetrazolium (MTS; Sigma, St. Louis, MO, USA) staining. Viable cancer tissues were weighed on a chemical balance and placed on the prepared collagen gel surfaces (Gelfoam ${ }^{\circledR}$; Pharmacia \& Upjohn) in 96-well plates (Figure 2). 
A

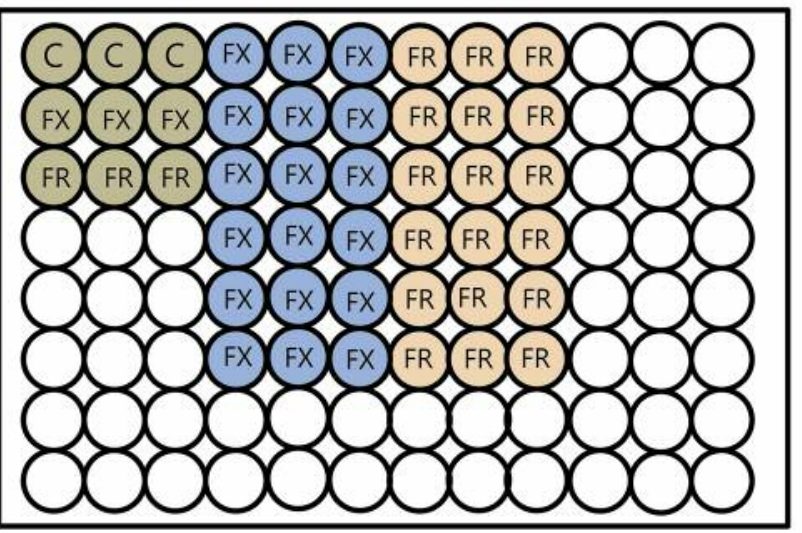

B

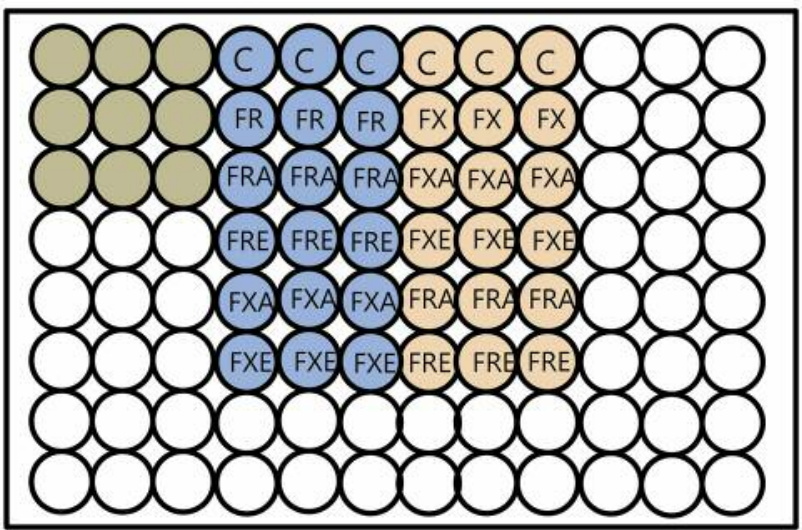

Figure 2. Arrangement of a 96-well plate for first- and second-line treatment regimens for metastatic colorectal cancer. $C$, Control; FX, 5-fluorouracil $(5-F U)+$ leucovorin + oxaliplatin; FR, 5-FU + leucovorin + irinotecan; FXA, FX + bevacizumab; FXE, FX + cetuximab; FRA, FR + bevacizumab; FRE, FR + cetuximab.

(ii) Tissue culture with first-line anticancer drugs. In this study, first-line chemotherapy regimens were confined to FX and FR. Three replicates were concurrently run for the control and treatment groups. The first-line anticancer drugs were dissolved in RPMI-1640 (Gibco) medium containing 10\% fetal bovine serum (FBS, Biowest, Nuaille, France) in a humidified atmosphere containing $95 \%$ air and $5 \% \mathrm{CO}_{2}$. The drug solutions were added to each well (Figure 2A), and the plates were incubated for 3 days at $37^{\circ} \mathrm{C}$.

(iii) Determination of sensitivity to first-line drugs. After histoculture, $900 \mu \mathrm{l}$ of HBSS containing $0.1-\mathrm{mg} / \mathrm{ml}$ collagenase (type I; Sigma) and $100 \mu \mathrm{l}$ of $0.2 \%$ MTT (Sigma) solution dissolved in phosphate-buffered saline was added to each culture well. Plates were incubated for another $4 \mathrm{~h}$, the medium removed, and $0.5 \mathrm{ml}$ of dimethyl sulfoxide (Sigma) was added to each well to extract MTT formazan. Extracts from each well $(100 \mu \mathrm{l})$ were transferred to a 96-well plate and the absorbance was measured at $540 \mathrm{~nm}$ using a microplate reader (VersaMax, Sunnyvale, CA, USA). The rate of inhibition of cancer-cell viability (IR) was calculated using the following equation: IR $(\%)=(1-$ mean absorbance of treated wells per gram of tumor/mean absorbance of control wells per gram of tumor) $\times 100$. The IR cutoff value for a positive response was previously determined to be $\geq 30 \%(11,12)$ and this was used the present study.

(iv) Tissue culture with second-line anticancer drugs. After the measurement of IR for first-line drug regimens (HDRA), the culture media and first-line drugs were removed. Three replicates were concurrently run for the control and treatment groups. Second-line anticancer drugs were dissolved in RPMI-1640 medium (Gibco) containing $10 \%$ FBS (Biowest) in a humidified atmosphere containing $95 \%$ air and $5 \% \mathrm{CO}_{2}$. The drug solutions were added to each well (Figure 2B), and the plates were incubated for another 2 days at $37^{\circ} \mathrm{C}$.

(v) Determination of sensitivity to second-line drugs. After the second histoculture, the IR for each drug regimen was measured in the same manner with an MTT end-point assay. An exemplary final ITRA report is shown in Figure 3.
Evaluation of tumor response. Each patient's response to treatment was assessed every three or four chemotherapy cycles with consistent imaging techniques such as computed tomography or magnetic resonance imaging. According to the Revised RECIST (Response Evaluation Criteria in Solid Tumors) guideline (version $1.1,2009$ ) (13), complete response (CR) was defined as the disappearance of all metastatic lesions and a partial response (PR) as a reduction of at least $30 \%$ of the sum of the longest diameter of metastatic lesions, with no evidence of new lesions. Progressive disease (PD) was defined as an increase by at least $20 \%$ of the sum of the longest diameters of either metastatic or recurrent lesions. Stable disease was reported when the criteria for either PR or PD were not met. The primary endpoint was a correlation between the ITRA results and the clinical response in response rate (RR); this was defined as positive for tumor responses of CR or PR.

Statistical analysis. A cross-table analysis employing Pearson's chisquare test or Fisher's exact test, as appropriate, was used to compare categorical variables. A $p$-value of 0.05 was considered statistically significant. All calculations were carried out using SPSS software (version 21.0; IBM Corp., Armonk, NY, USA).

\section{Results}

ITRA tumor growth-inhibition ratios. Of 120 evaluated samples, IR with first-line regimens for FR and FX were $36 \%$ and $32.6 \%$, respectively. IRs for second-line regimens were lower than those of first-line regimens (Figure 4A). The IR for firstline FR was significantly better than that for FX $(p<0.001)$. The differences in IRs between first- and second-line regimens were significant for FR $(p<0.001)$, but not for $\mathrm{FX}(p=0.077)$.

For second-line treatment, switching from FX to FR or from FR to FX and adding a targeted agent resulted in better IRs than adding a targeted drug to the first-line regimen $(p<0.001)$. In terms of targeted agents, adding cetuximab to second-line 

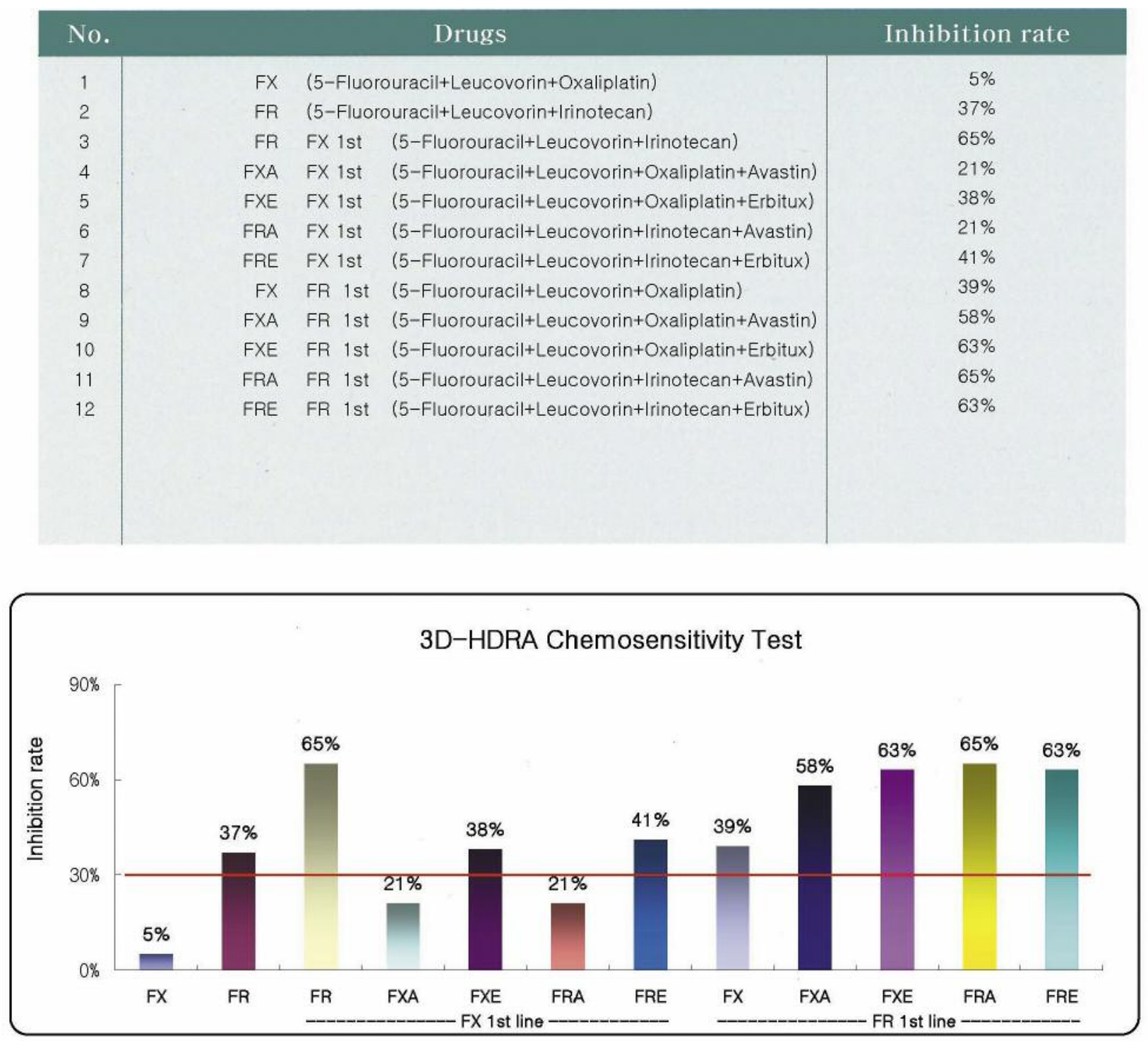

Figure 3. Example of a report for an integrated tumor response assay of responsiveness of colorectal tumor samples to different chemotherapy regimens.

regimens resulted in significantly better IR than adding bevacizumab $(p<0.001)$. The percentages of chemosensitive tumors were similar for each regimen (Figure 4B).

Clinical correlation of HDRA and ITRA. The clinical responses of 120 patients treated with first-line regimens are summarized in Table III. The overall RR was $50 \%$. On repeated assessments, however, RR decreased. Out of 79 HDRAs of first-line chemotherapeutic regimens, the correlation with patient clinical response was $70.9 \%(56 / 79)$, with $73.2 \%$ (30/41) true-positives and $68.4 \%$ (26/38) truenegatives. The sensitivity was $71.4 \%$ (30/42) and specificity
$70.3 \%$ (26/37). For the 42 patients completing second-line chemotherapy, the accuracy of ITRA for predicting response to second-line chemotherapy was $61.9 \%$ (Table IV).

We analyzed clinicopathological parameters that might predict better chemoresponsiveness to first- and second-line chemotherapy. Microsatellite instability $(p=0.03)$, poorly differentiated or mucinous histology $(p=0.005)$, and large tumor (size $>6 \mathrm{~cm}, p=0.046$ ) were significantly associated with poor chemoresponsiveness to first-line chemotherapy. Lymphovascular invasion was significantly related to poor chemoresponsiveness to second-line chemotherapy $(p=0.049)$. However, multivariate analysis did not demonstrate any 

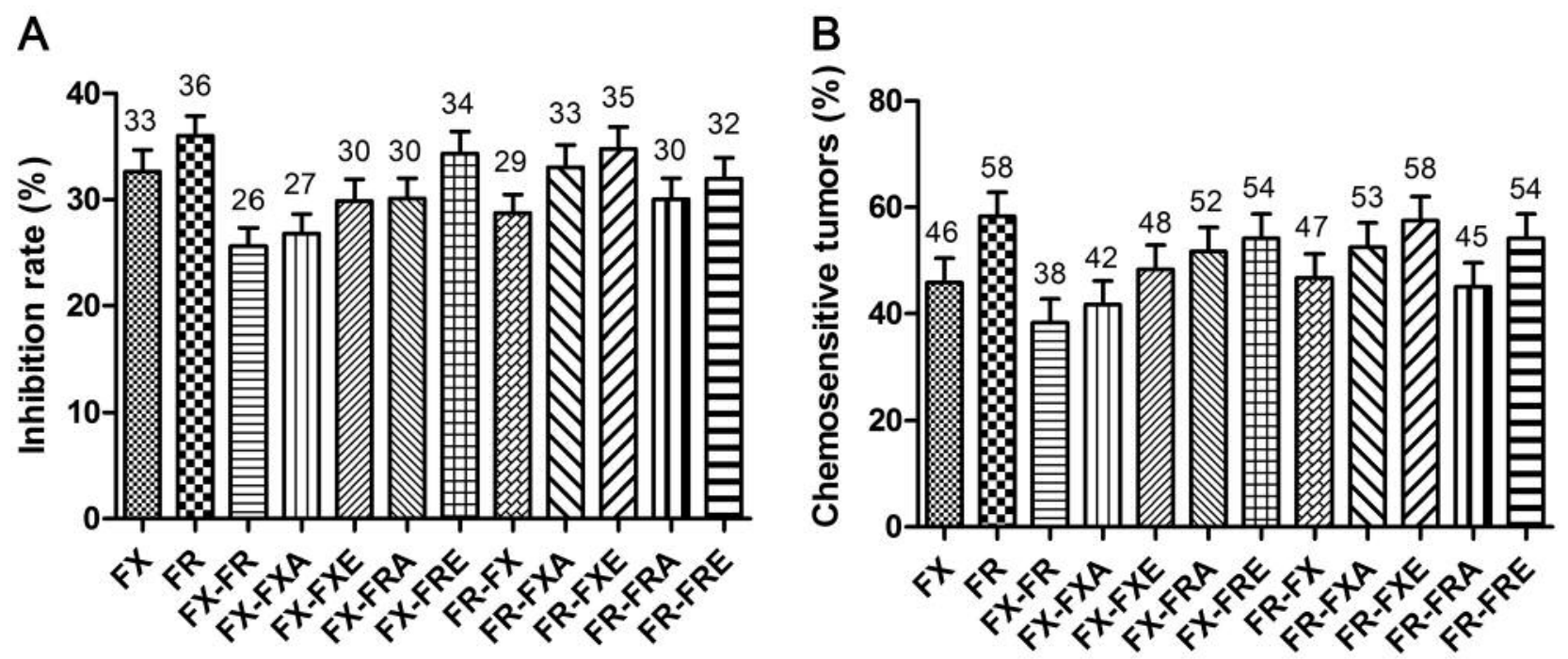

Figure 4. A: Tumor cell inhibition rates of established regimens for metastatic colorectal carcinoma. B: Proportion of chemosensitive tumors according to treatment regimen. FX, 5-Fluorouracil $(5-F U)+$ leucovorin + oxaliplatin; $F R, 5-F U+$ leucovorin + irinotecan; FXA, FX + bevacizumab; FXE, FX + cetuximab; FRA, FR + bevacizumab; FRE, FR + cetuximab. Data are reported as means \pm SEM.

Table III. Treatment response to first- and second-line chemotherapy among patients with colorectal carcinoma.

\begin{tabular}{lccc}
\hline Clinical response & Total $(\mathrm{n}=120), \mathrm{n}(\%)$ & First-line $(\mathrm{n}=79), \mathrm{n}(\%)$ & Second-line $(\mathrm{n}=42), \mathrm{n}(\%)$ \\
\hline CR & $3(2)$ & 2 & 1 \\
PR & $57(47)$ & 40 & 17 \\
SD & $41(34)$ & 26 & 15 \\
PD & $20(17)$ & 11 & 9 \\
Response rate & $50 \%$ & $53 \%$ & $43 \%$ \\
\hline
\end{tabular}

CR, Complete response; PR, partial response; $\mathrm{SD}$, stable disease; $\mathrm{PD}$, progressive disease.

significant predictor of chemoresponse to first- or second-line chemotherapy. Furthermore, clinicopathological parameters were not correlated with the accuracy of ITRA or HDRA.

\section{Discussion}

Pathways of inherent and acquired resistance account for the high rate of anticancer treatment failure. The mechanisms or pathways mediating resistance are explained by changes in intratumoral drug exposure or failure to elicit cytotoxicity (14). In terms of acquired resistance, sufficient exposure to a specific anticancer drug can elicit drug resistance through various pathways. In order to simulate the cellular environment in patients with recurrent CRC, we examined tumor cells that had survived first-line chemotherapy regimens, culturing them with second-line regimens. Our hypothesis on which ITRA was based was that tumor cells surviving after chemotherapy in our in vitro assay had equivalent genetic traits to those of in vivo recurrent CRC.
Table IV. Correlation between integrative tumor-response assay (ITRA) and clinical response to second-line chemotherapy in patients with colorectal carcinoma.

\begin{tabular}{lccc}
\hline & \multicolumn{3}{c}{ Clinical response, $\mathrm{n}$} \\
\cline { 2 - 4 } ITRA, $\mathrm{n}$ & Positive* $^{*}$ & Negative & Total \\
\hline & & & \\
Positive & 8 & 6 & 14 \\
Negative & 10 & 18 & 28 \\
Total & 18 & 24 & 42 \\
\hline
\end{tabular}

Sensitivity: $44.4 \%$ (8/18); specificity: $75 \%$ (18/24); true-positive rate: $57.1 \%(8 / 14)$; true-negative rate: $64.3 \%$ (18/28); tccuracy: $61.9 \%$ (26/42); *complete response or partial response.

Although it was difficult to prove this, our results with ITRA showed a slightly positive correlation with clinical response to second-line chemotherapy. 
ITRA is not simply a repetition of HDRA. It examines the sensitivity of resistant tumor cells. HDRA requires an increased volume of tumor sample when a greater number of chemotherapy regimens are evaluated. In the present study, we evaluated a total of twelve regimens of combination chemotherapy for all specimens, compared with seven regimens examined by HDRA in our previous work (5). Because ITRA used the same specimen volume $\left(1 \mathrm{~cm}^{3}\right)$ as HDRA, we were unable to perform ITRA in nine cases in the early period of this study. The specimen volume must be adjusted to the number of treatment regimens evaluated. Thus, a limitation of ITRA is that it cannot be performed in cases with small tumors that yield insufficient specimens.

Our twelve chemotherapy regimens were chosen considering every possible sequential combination of agents, assuming that targeted agents were used for second-line chemotherapy. Compared to the effort involved, the efficiency of ITRA was low in cases using targeted agents (6/42). As conditions vary in clinical practice, sequential combinations of chemotherapy regimens to be assayed can be adjusted. At the end of enrollment of the present trial, the Korean government extended economic support for targeted agents, allowing their use as first-line chemotherapy in patients with metastatic CRC. After that, we updated our assay to test seventeen possible sequential combinations of chemotherapy, any of which might be used in clinical practice. In terms of assay efficiency, ITRA might be most useful for the combination chemotherapy regimens of FX and FR without targeted agents.

The monoclonal antibody cetuximab, which targets the epidermal growth factor receptor (EGFR), has expanded the range of treatment options for metastatic CRC.(15) EGFR and downstream signaling pathways are activated by several mechanisms, including overexpression of the receptor, overexpression of the ligand, activation by a receptor mutation, or inactivation of tumor-suppressor genes (16). The presence of wild-type V-Ki-ras2 Kirsten rat sarcoma viral oncogene homolog (KRAS) is considered a predictor of response to EGFR inhibitor therapy $(17,18)$. The presence of a KRAS mutation is important in choosing the appropriate targeted agent, especially for antiEGFR therapy (19). It may be difficult to use cetuximab according to ITRA results in the presence of mutant form of KRAS. Bevacizumab is a monoclonal antibody against vascular endothelial growth factor (VEGF) and has shown clinical activity against metastatic CRC (20). Although our threedimensional culture system has the advantage of more accurately reflecting the in vivo microenvironment, the performance of ITRA in vitro is difficult to compare with the in vivo cellular environment as it cannot account for vascular supply. Thus, the value of ITRA for evaluating response to bevacizumab is questionable because angiogenesis cannot be replicated in the in vitro cell-culture system. This may explain why ITRA was less successful in predicting response to the addition of bevacizumab than to that of cetuximab as second-line chemotherapy.
The RRs to first-line chemotherapy with FX and FR for patients with metastatic CRC are 40-50\%, which further decrease with subsequent treatment (21). Recent combined chemotherapy using anti-EGFR and anti-VEGF agents enhanced RRs of chemotherapy and improved survival up to 30 months for patients with metastatic CRC (22). Although our ITRA was not highly predictive of response to these agents, it might be helpful in choosing optimal chemotherapy regimens and improving survival of patients with metastatic CRC.

This study has several limitations. We were only able to perform ITRA in one-third of the initially enrolled patients. Reasons for this varied, including patient choice, economic status, whether medical insurance covered the anticancer drugs, and an inadequate sequence of chemotherapy which was different from that of our study protocol. In addition, although ITRA results were positively correlated with clinical drug responses, the accuracy was relatively low. This might be because of the complexity of chemotherapy regimens, low drug concentrations, or a low cutoff value for IR. This is a fundamental limitation of an in vitro assay, as we suggested in our previous investigation of HDRA (5). Furthermore, an innate limitation of ITRA is its inability to test circulating tumor cells or cancer stem cells, which may be particularly resistant to different chemotherapeutic agents (23).

In conclusion, despite its relatively low accuracy, ITRA may be a feasible and useful technique for predicting therapeutic efficacy and selecting appropriate first-line and second-line anticancer regimens for individual patients with metastatic CRC.

\section{Acknowledgements}

This study was supported by grants from the Asan Institute for Life Sciences (2014-596), the Korea Research Foundation (2013R1A2A1A03070986), Ministry of Science, ICT, and Future Planning, the Korea Health 21 R\&D Project (HI06C0868 and HI13C1750), and the Center for Development and Commercialization of Anti-Cancer Therapeutics (A102059), Ministry of Health and Welfare, Republic of Korea.Seoul, Korea.

The Authors would like to thank Enago (http://www.enago.co.kr) for English language review.

\section{References}

1 Kim JB, Stein R and O'Hare MJ: Three-dimensional in vitro tissue culture models of breast cancer - a review. Breast Cancer Res Treat 85: 281-291, 2004.

2 Robbins KT, Connors KM, Storniolo AM, Hanchett C and Hoffman RM: Sponge-gel-supported histoculture drug-response assay for head and neck cancer. Correlations with clinical response to cisplatin. Arch Otolaryngol Head Neck Surg 120: 288-292, 1994.

3 Furukawa T, Kubota T and Hoffman RM: Clinical applications of the histoculture drug response assay. Clin Cancer Res 1: 305$311,1995$. 
4 Hasegawa Y, Goto M, Hanai N, Ijichi K, Adachi M, Terada A Hyodo I, Ogawa T and Furukawa T: Evaluation of optimal drug concentration in histoculture drug response assay in association with clinical efficacy for head and neck cancer. Oral Oncol 43: 749-756, 2007.

5 Yoon YS, Kim CW, Roh SA, Cho DH, Kim GP, Hong YS, Kim TW, Kim MB and Kim JC: Applicability of histoculture drug response assays in colorectal cancer chemotherapy. Anticancer Res 32: 3581-3586, 2012.

6 Cook AD, Single R and McCahill LE: Surgical resection of primary tumors in patients who present with stage IV colorectal cancer: an analysis of surveillance, epidemiology, and end results data, 1988 to 2000. Ann Surg Oncol 12: 637-645, 2005.

7 Yoon YS, Kim CW, Lim SB, Yu CS, Kim SY, Kim TW, Kim MJ and Kim JC: Palliative surgery in patients with unresectable colorectal liver metastases: a propensity score matching analysis. J Surg Oncol 109: 239-244, 2014.

8 Yamashita K, Urakami A, Kubozoe T, Ikeda M, Hirabayashi Y, Yamamura M, Iki K, Akiyama T, Matsumoto H, Hirai T, Sadahira $\mathrm{Y}$ and Tsunoda $\mathrm{T}$ : In vitro detection of cross-resistant and non-cross-resistant agents with fluorouracil for patients with colorectal cancer. Int J Clin Oncol 10: 328-332, 2005.

9 Andreotti PE, Cree IA, Kurbacher CM, Hartmann DM, Linder D, Harel G, Gleiberman I, Caruso PA, Ricks SH, Untch M, Sartori C and Bruckner HW: Chemosensitivity testing of human tumors using a microplate adenosine triphosphate luminescence assay: clinical correlation for cisplatin resistance of ovarian carcinoma. Cancer Res 55: 5276-5282, 1995.

10 Vescio RA, Redfern CH, Nelson TJ, Ugoretz S, Stern PH and Hoffman RM: In vivo-like drug responses of human tumors growing in three-dimensional gel-supported primary culture. Proc Natl Acad Sci USA 84: 5029-5033, 1987.

11 Kim JC, Kim DD, Lee YM, Kim TW, Cho DH, Kim MB, Ro SG, Kim SY, Kim YS and Lee JS: Evaluation of novel histone deacetylase inhibitors as therapeutic agents for colorectal adenocarcinomas compared to established regimens with the histoculture drug response assay. Int J Colorectal Dis 24: 209218, 2009.

12 Kim JC, Shin ES, Kim CW, Roh SA, Cho DH, Na YS, Kim TW, Kim MB, Hyun YL, Ro S, Kim SY and Kim YS: In vitro evaluation of histone deacetylase inhibitors as combination agents for colorectal cancer. Anticancer Res 29: 3027-3034, 2009.

13 Eisenhauer EA, Therasse P, Bogaerts J, Schwartz LH, Sargent D, Ford R, Dancey J, Arbuck S, Gwyther S, Mooney M, Rubinstein L, Shankar L, Dodd L, Kaplan R, Lacombe D and Verweij J: New response evaluation criteria in solid tumours: revised RECIST guideline (version 1.1). Eur J Cancer 45: 228247, 2009.

14 Mellor HR and Callaghan R: Resistance to chemotherapy in cancer: a complex and integrated cellular response. Pharmacology 81: 275-300, 2008.
15 Silvestri A, Pin E, Huijbers A, Pellicani R, Parasido EM, Pierobon M, Petricoin E, Liotta L and Belluco C: Individualized therapy for metastatic colorectal cancer. J Intern Med 274: 1-24, 2013.

16 Siena S, Sartore-Bianchi A, Di Nicolantonio F, Balfour J and Bardelli A: Biomarkers predicting clinical outcome of epidermal growth factor receptor-targeted therapy in metastatic colorectal cancer. J Natl Cancer Inst 101: 1308-1324, 2009.

17 Van Cutsem E, Kohne CH, Hitre E, Zaluski J, Chang Chien CR, Makhson A, D'Haens G, Pinter T, Lim R, Bodoky G, Roh JK, Folprecht G, Ruff P, Stroh C, Tejpar S, Schlichting M, Nippgen $\mathrm{J}$ and Rougier P: Cetuximab and chemotherapy as initial treatment for metastatic colorectal cancer. N Engl J Med 360: 1408-1417, 2009.

18 Bokemeyer C, Bondarenko I, Makhson A, Hartmann JT, Aparicio J, de Braud F, Donea S, Ludwig H, Schuch G, Stroh C, Loos AH, Zubel A and Koralewski P: Fluorouracil, leucovorin, and oxaliplatin with and without cetuximab in the first-line treatment of metastatic colorectal cancer. J Clin Oncol 27: 663-671, 2009.

19 Lievre A, Bachet JB, Boige V, Cayre A, Le Corre D, Buc E, Ychou M, Bouche O, Landi B, Louvet C, Andre T, Bibeau F, Diebold MD, Rougier P, Ducreux M, Tomasic G, Emile JF, Penault-Llorca $\mathrm{F}$ and Laurent-Puig P: KRAS mutations as an independent prognostic factor in patients with advanced colorectal cancer treated with cetuximab. J Clin Oncol 26: 374-379, 2008.

20 Hurwitz H, Fehrenbacher L, Novotny W, Cartwright T, Hainsworth J, Heim W, Berlin J, Baron A, Griffing S, Holmgren E, Ferrara N, Fyfe G, Rogers B, Ross R and Kabbinavar F: Bevacizumab plus irinotecan, fluorouracil, and leucovorin for metastatic colorectal cancer. N Engl J Med 350: 2335-2342, 2004.

21 Sabharwal A and Kerr D: Chemotherapy for colorectal cancer in the metastatic and adjuvant setting: past, present and future. Expert Rev Anticancer Ther 7: 477-487, 2007.

22 Yaffee P, Osipov A, Tan C, Tuli R and Hendifar A: Review of systemic therapies for locally advanced and metastatic rectal cancer. J Gastrointest Oncol 6: 185-200, 2015.

23 Lim SH, Becker TM, Chua W, Ng WL, de Souza P and Spring KJ: Circulating tumour cells and the epithelial-mesenchymal transition in colorectal cancer. J Clin Pathol 67: 848-853, 2014.

Received December 24, 2016

Revised February 4, 2017

Accepted February 8, 2017 